\title{
Research Paper \\ Quality of Life and Its Related Factors among Elderly with Diabetes
}

\author{
Vahidreza Borhaninejad ${ }^{1}$, Leila Kazazi², Marjan Haghi², *Negin Chehrehnegar ${ }^{2}$
}

1. Research Center for Infectious and Tropical Disease, Kerman University of Medical Sciences, Kerman, Iran.

2. Iranian Research Center on Ageing, University of Social Welfare and Rehabilitation Sciences, Tehran, Iran.

Citation: Borhaninezhad VR, Kazazi L, Haghi M, Chehrehnegar N. [Quality of Life and Its Related Factors among Elderly with Diabetes (Persian)]. Iranian Journal of Ageing. 2016; 11(4):162-173. http://dx.doi.org/10.21859/sija-1101162

: http://dx.doi.org/10.21859/sija-1101162

Received: 11 Oct. 2015 Accepted: 13 Feb. 2016

\section{ABSTRACT}

Objectives Diabetes is a common chronic disease in the elderly that affects their quality of lives. As "quality of life" is one of the most important factors and indicators of health, this study examined the "quality of life" in the diabetic elderly people.

Methods \& Materials This descriptive-analytic study was conducted on diabetic elderly people living in Kerman, Iran, in 2013. A total of 120 patients were randomly selected with multistage sampling method. For data collection, the demographic and SF-36 questionnaires were used. Then, the obtained data were analyzed by SPSS version 18 by conducting t-test, correlation coefficient, and analysis of variance (ANOVA).

Results The results showed that the mean age of the subjects was 71.5 years. The total "quality of life" score of diabetic patients was 46.48 . The elderly showed that the highest mean among the 8 dimensions of "quality of life" in physical health dimension belonged to general health (47.87), and with regard to mental health dimension, the highest score was in social function (49.78). The total score of physical dimension was 44.89 , and the total score of mental dimension was 48.07 . Furthermore, the results of t-test and 1-way ANOVA indicated that there were significant correlations between "quality of life" and variables of sex, educational level, complications of diabetes, income, age, duration of diabetes, and body mass index $(\mathrm{P} \leq 0.05)$.

Conclusion In our society, no normative standard has been set for the "quality of life." However, the mean value of 50 with standard deviation of 10 can be an acceptable and standard score for the "quality of life" among the elderly. The mean score of "quality of life" among diabetic elderly people in this study was 46.48 which was lower than our standard. Also, in studying the 8 dimensions of "quality of life," the mean score of all dimensions was lower than 50 . Prevalence of chronic diseases like diabetes among the older people could end in lowering the mean score of physical dimensions as compared to mental dimensions of "quality of life." Something that was mentioned in the present study, too.The lower quality of life among women could be due to gender inequality in using health system. As a result, the women are more vulnerable to problems as well as mental and physical diseases which decreases their quality of lives. The people with higher education, armed with their health knowledge and using self-care methods provide the grounds for increasing their quality of lives.As regards the increasing rate of diabetic patients in ageing population and considering that chronic diseases affect and lower physical and mental aspects of "quality of life" in older people, and in line with improving the "quality of life" in these people, we suggest that policymakers and authorities pay proper attention to physical, mental, and social support of this group.
Key words: Quality of life, Elderly, Diabetes

\footnotetext{
……
}

* Corresponding Author:

Negin Chehrehnegar, PhD Candidate

Address: Iranian Research Center on Ageing, University of Social Welfare and Rehabilitation Sciences, Kodakyar Ave., Daneshjo Blv., Evin, Tehran, Iran. Tel: +98 (912) 6836728

E-mail: negin.chehrenegar@gmail.com 


\title{
بررسى كيفيت زندكى سالمندان ديابتى شهر كرمان و عوامل مرتبط با آن در سال بوس
}

\author{
وحيدرضا برهانىنزراد'، ليلا كزازى'، مرجان حقى'، "نكين ههرونكار؟ \\ 1- مركز تحقيقات بيمارىهاى عفونى و كرمسيرى، دانشكاه علوم يزشكى كرمان، كرمان، ايران. \\ r- بمركز تحقيقات سالمندى ايران، انشكَاه علوم برزيستى و توانبخشى، تهران، ايران.
}

\begin{abstract}
حكبد

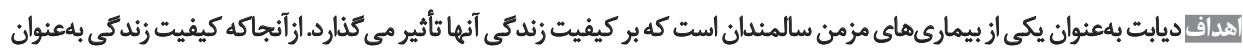

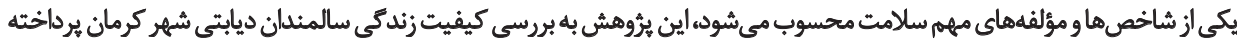

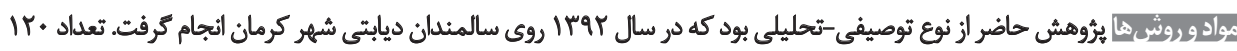

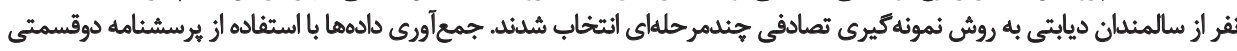

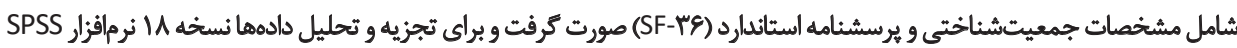

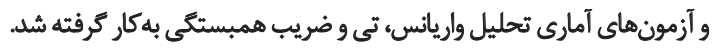

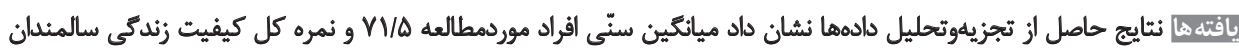

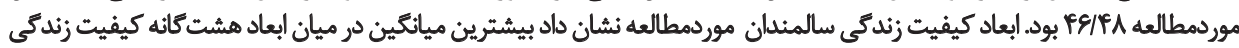

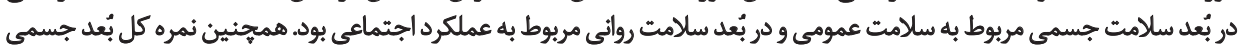

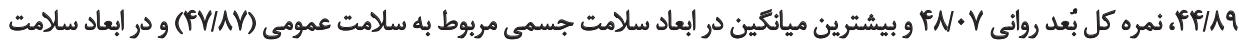

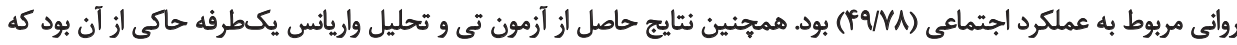

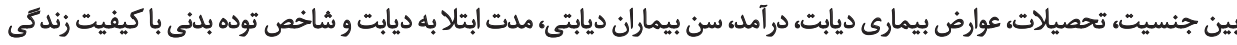

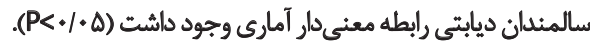

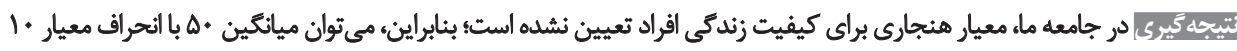

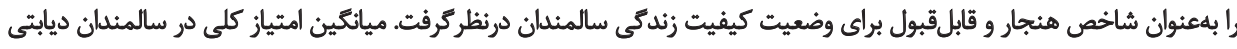

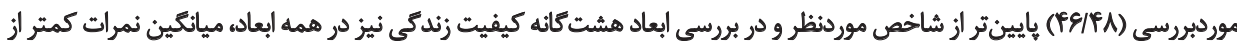

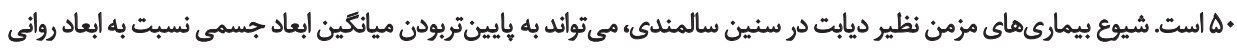

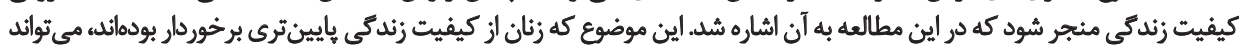

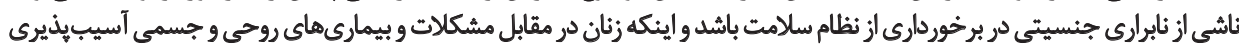

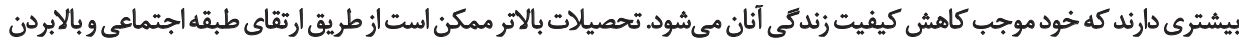

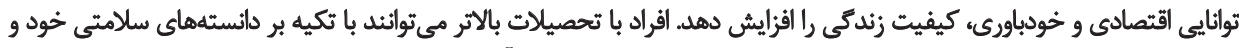

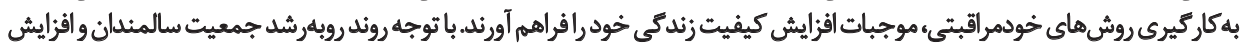

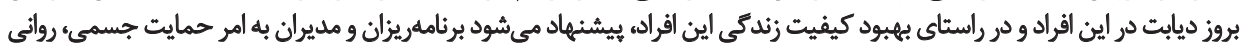

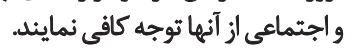

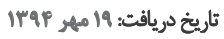

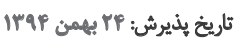

كليدواثهها:

كيفيت زئدكى، سالمندانء ديابت

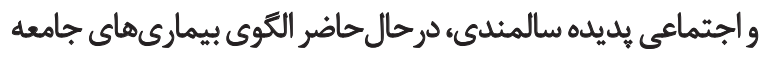

مقدمه

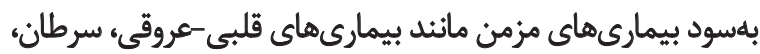

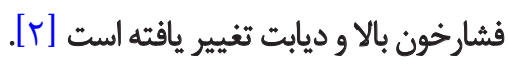

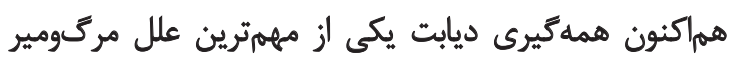

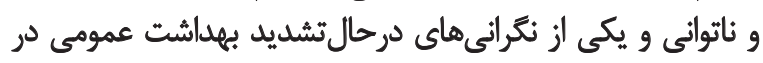

بهبود شرايط زندكى و افزايش طول عمر و اميدبهزندگىى، بديده

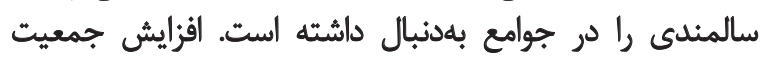

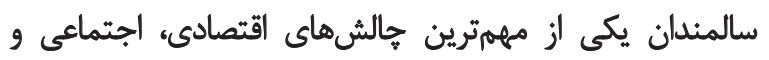

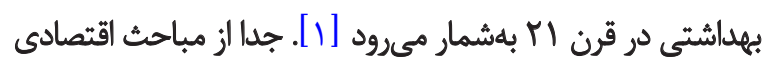


شايان ذكر است در زمينه اهميت مطالعات كيفيت زندكى و رشد

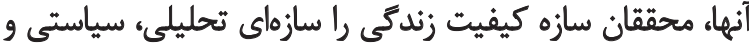

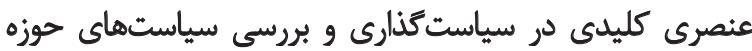

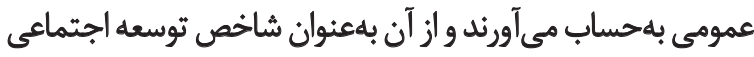

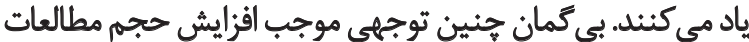

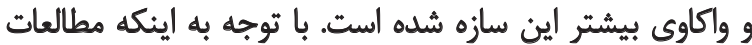

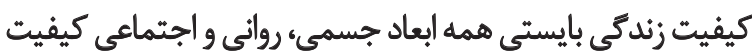

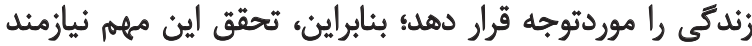

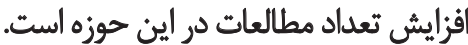

ازيكسو، جندبُعدىبودن سازه كيفيت زندكى امكان ورود

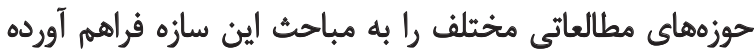

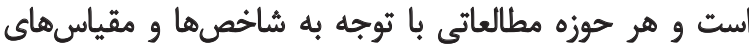

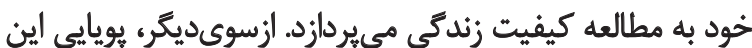

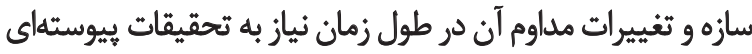

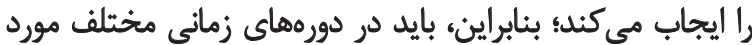

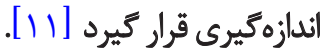

در اين بين كيفيت زندگى سالمندان و افراد ديابتى نيز در

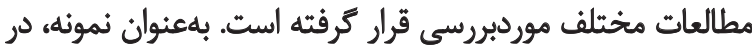
مطالعهاي كه توسط ارسطو و همكاران در شهر تهرئف

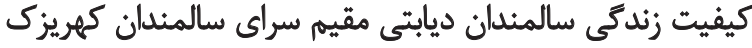

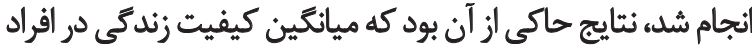

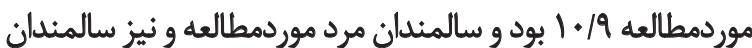

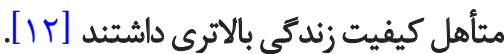
در مطالعه نجاتى و همكاران در شهر كاشان، سطح كيفيت زندگى

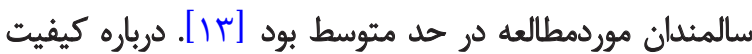

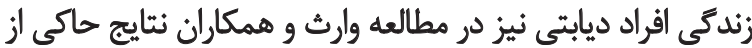

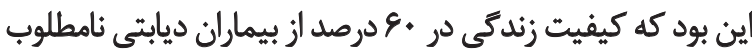

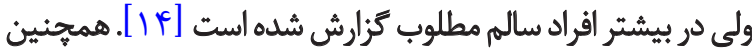

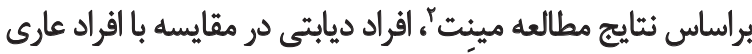

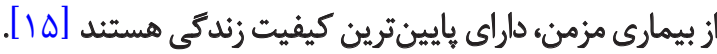
همانطوركه اشاره شد تحقيقات مختلف نشان ميدهد كئ هي هم

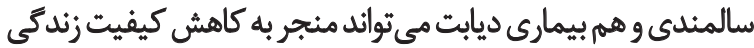

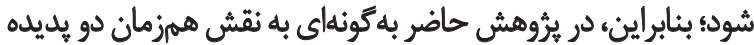

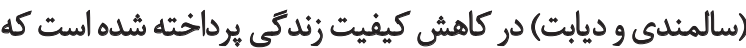
مى تواند اين مطالعه را از ديكر مطالعات مشابه متمايز نمايد.

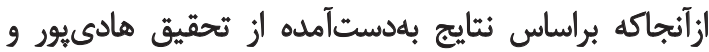

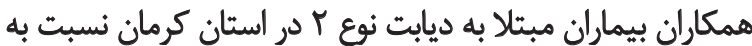

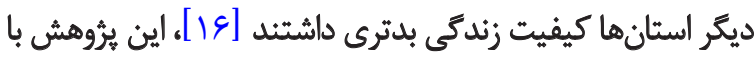

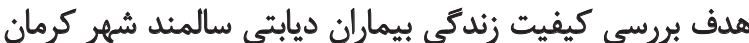

دئياست. براساس روند فعلى، حدود VD درصد موارد ديابتى دنيا در

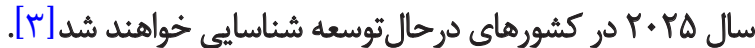

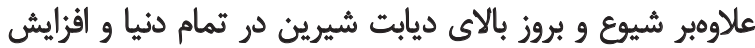

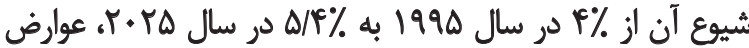

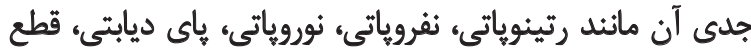

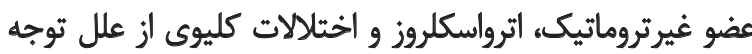

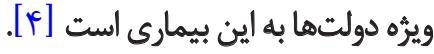

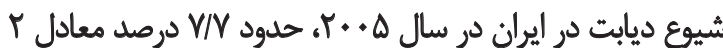

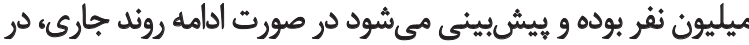

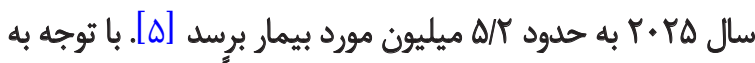

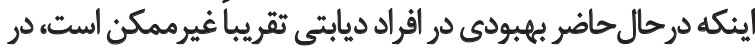

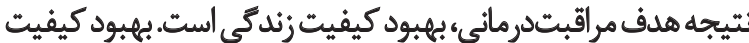

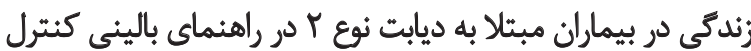

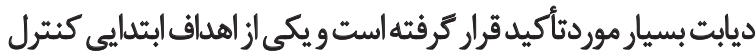

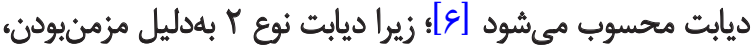

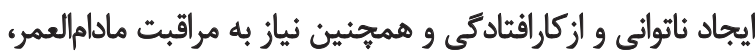

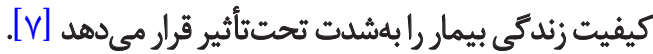
كيفيت زندگى به آن دسته از ويرگكىهاى تعريفشده مي كويند

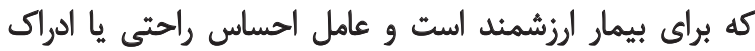

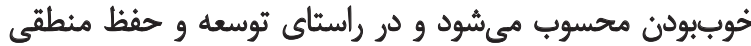

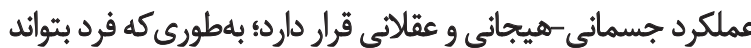

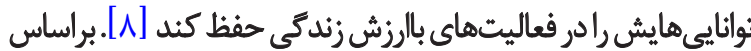
تعريف سازمان جهانى بهداشت در سال

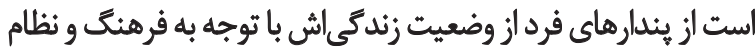

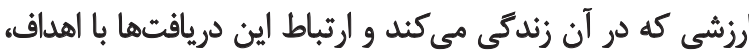

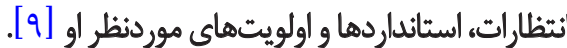

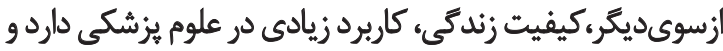

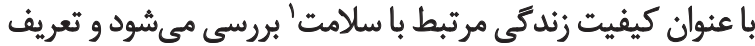

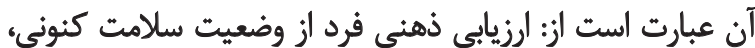

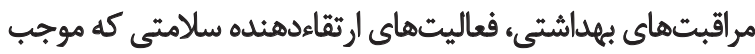

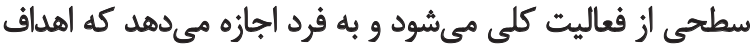

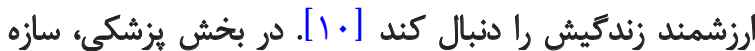

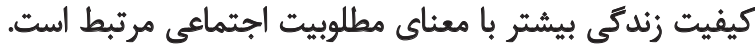

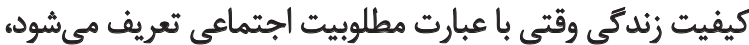

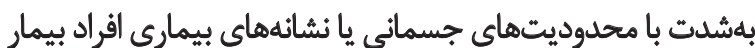

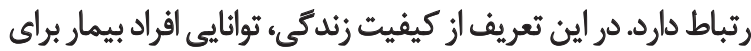

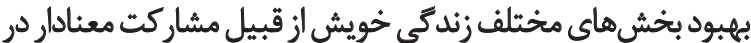

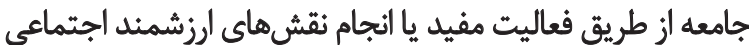

$$
\text { مورد بررسى قرار مى كيرد [1 إن] }
$$




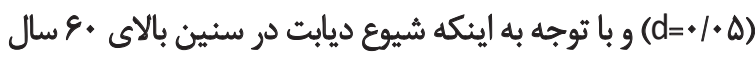

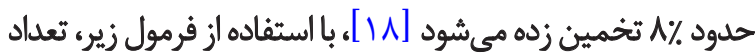

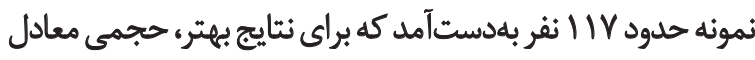

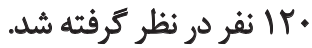

دادهها به كمك نسخه 1 انرمافزار SPSS و آزمونهاى آمارى

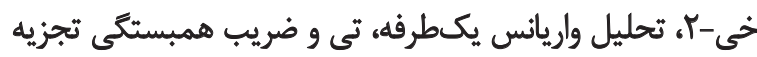

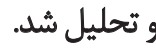

يافتهها

نتايج حاصل از تجزيه و تحليل دادهها نشان داد كه ميانكين

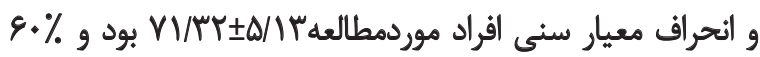

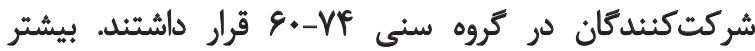

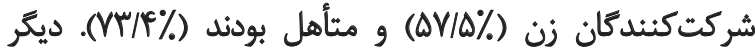
اطلاعات جمعيتشناختى در جدول شماره إن أذكر شده است. ابعاد كيفيت زندكى سالمندان موردمطالعه در جدول شماره

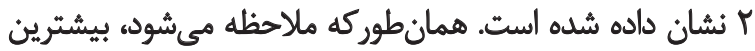

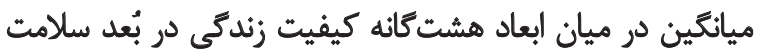

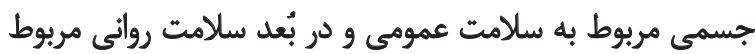

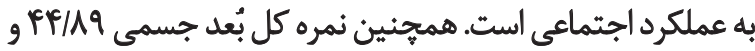

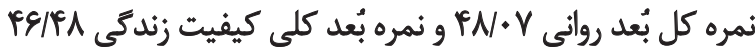

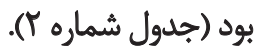

\section{نتايج حاصل از آزمون تى و تحليل واريانس يكسطرفه حاكى از آن آن}

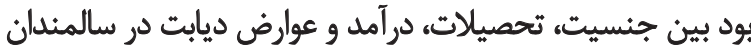

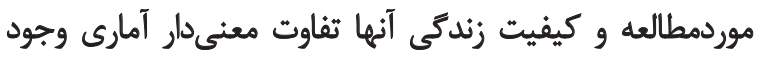

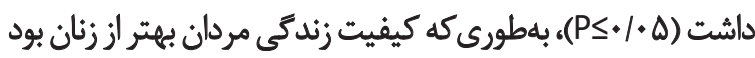

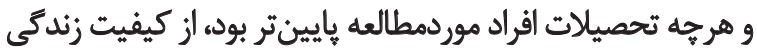

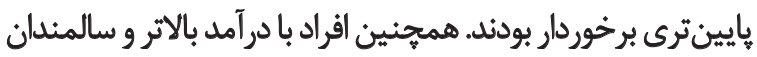

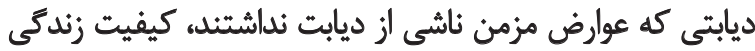

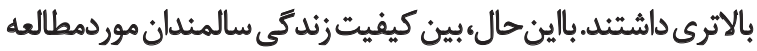

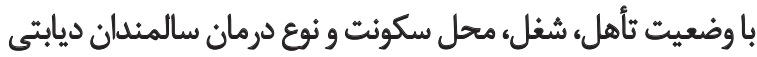

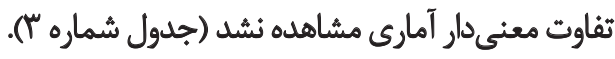

آرمون ضريب همبستكى يُيرسون نشان داد بين سن سالمندان

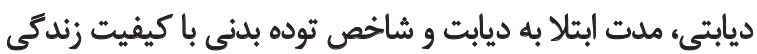

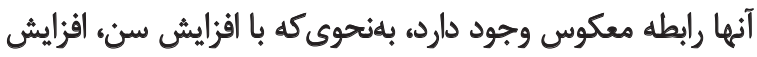

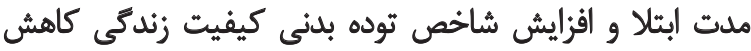

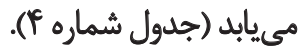

بحث

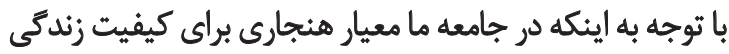

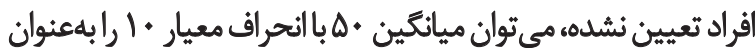

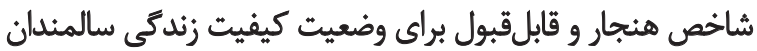

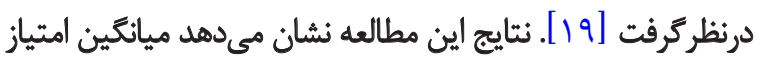

و ارتباط آن با عواملى نظير نحوه درمان، طول مدت ديابت،

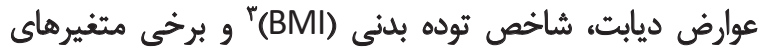

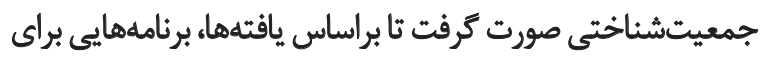

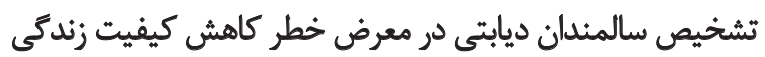

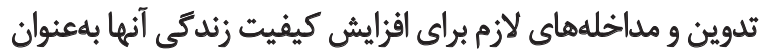
يكى از اهداف كنترل ديابت انجام شود.

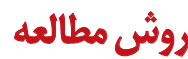

مطالعه حاضر يك مطالعه توصيفى -تحليلى و از نوع مقطعى بود

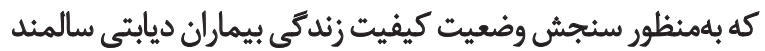

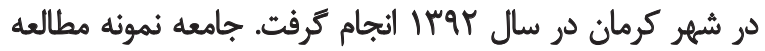

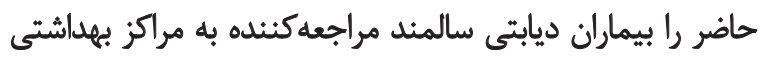

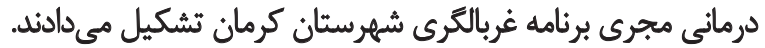

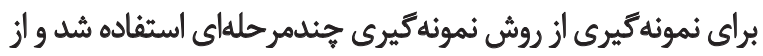

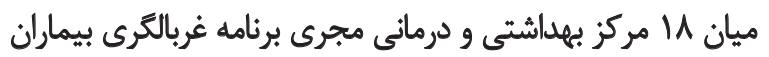

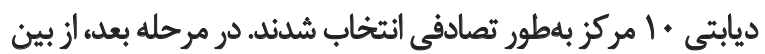

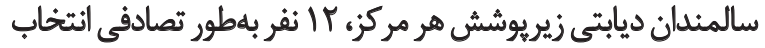

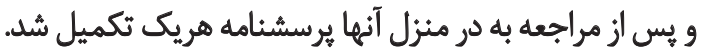

$$
n=\frac{z_{1-\alpha / 2}^{2} \times P \times(1-P)}{d^{2}}
$$

براى جمعآورى دادهها برسشنامه دوقسمتى شامل مشخصات

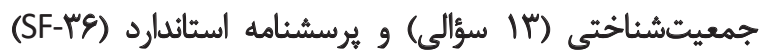

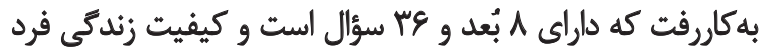

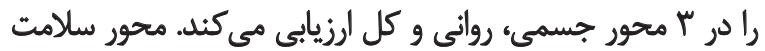

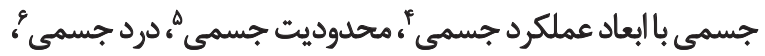

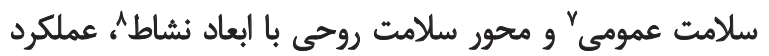

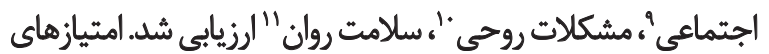

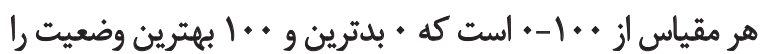

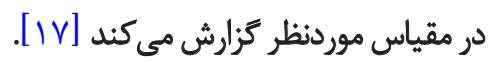
در حال حاضر اين ابزار بركاربردترين ابزار اندازمكيرى كيفيت

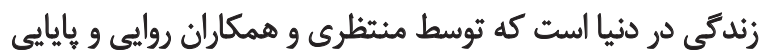

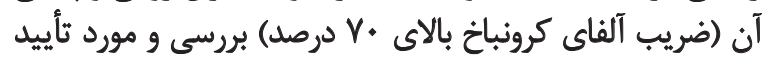

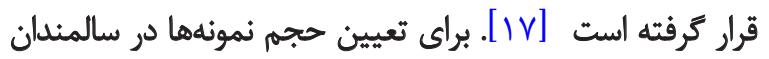

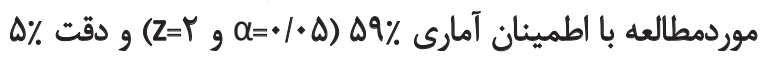

3. Body Mass index

4. Physical function

5. Physical role

6. Bodily pain

7. General health

8. Vitality

9. Social function

10. Role emotional

11. Mental health 
جدول ا. توزيع فراوائى ويرُكى ها جمعيتشُئاختى.

\begin{tabular}{|c|c|c|c|}
\hline درصد فراوانى & فراوانى & $\log 5$ & مثتيرها \\
\hline $9 . \%$ & $n$ & $q_{*}-h^{f}$ & \\
\hline$r . \%$ & rq & $V \Delta-q$. & سن \\
\hline $1 . \%$ & ir & +9 & \\
\hline$\Delta V / \Delta \%$ & $8 q$ & نj & \\
\hline$F r / \Delta \%$ & (1) & مرد & جنس \\
\hline $\begin{array}{l}\text { rele\% } \\
\boldsymbol{r}^{\prime} / \mathrm{r} \%\end{array}$ & $\begin{array}{l}M \\
M\end{array}$ & مترأهل & وضعيت تأهل \\
\hline $\begin{array}{l}\text { A) } / 8 \% \\
\text { WF\% }\end{array}$ & $\begin{array}{l}u \\
r\end{array}$ & روسير & هحل سكونت \\
\hline $11 / 8 \%$ & if & لبى بواد & \\
\hline rNM\% & $M$ & خواندنونوثشن & \\
\hline$r V / \Delta \%$ & rr & إتثائى & تحصيلات \\
\hline T०\% & r. & متونطb & \\
\hline$V / \Delta \%$ & 9 & دييلم و بالاتر & \\
\hline$ه \%$ & 8 & كارمند & \\
\hline $1 . \%$ & ir & آزاد & \\
\hline$\sim T / \Delta \%$ & D) & خانهدار & وضعيت الشتغال \\
\hline$M / \Delta \%$ & $r q$ & بازئشسته & \\
\hline $1 . \%$ & ir & ساير & \\
\hline$\Delta \cdot 1 / \%$ & 81 & كمتر از ه.مه & \\
\hline$m \in / r \%$ & il & 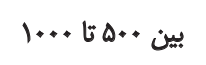 & درآمد \\
\hline$V / \Delta \%$ & 9 & 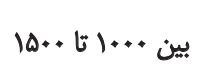 & (هزار تومان) \\
\hline$\Delta / \Lambda \%$ & $\checkmark$ & بالاي ..10 & \\
\hline sV/a $\%$ & A) & قرص & \\
\hline IV/a $\%$ & $r$ & أنسولين & نوع درمان \\
\hline $10 \%$ & $M$ & هر دو & \\
\hline
\end{tabular}

L

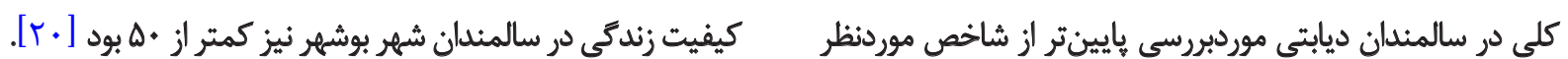
در مطالعه نجاتى و همكاران در شهر كاشان، سطح كيفيت درني

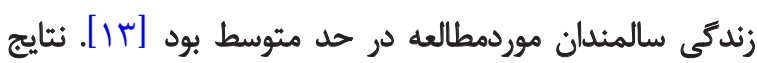

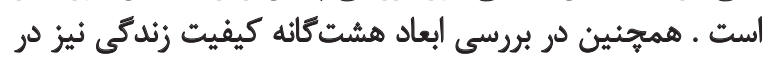

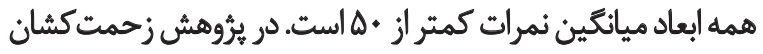

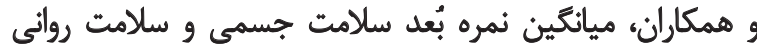


جدول r. ميانكين و انحراف ابعاد هشت كائه كيفيت بيماران ديابتى موردبررسى.

\begin{tabular}{|c|c|}
\hline ميانكين و انحراف معيار & ابعاد كيفيت زندكى \\
\hline FE/TI(TFIQ) & عملكرد جسمى \\
\hline RE/Tr(MTT) & هحدوديت جسمى \\
\hline PITKA(IQ/RT) & درد جسمي \\
\hline $\operatorname{PV/AV}(T \& / I Q)$ & سلامت عمومى \\
\hline$F \Delta / / Q(T T / M T)$ & 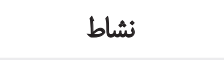 \\
\hline Pq/NA(IV/II) & عملكرد اجتماعى \\
\hline$e q / \pi f(1 q / / V)$ & مشكلات روحى \\
\hline FNIF(YNIQ) & سلامت روان \\
\hline$P \varphi / A q(Y) / M)$ & ابعاد جسمى \\
\hline$F N \cdot Y(19 / F V)$ & ابعاد روائى \\
\hline$F \in / T A(T \cdot / T \Delta)$ & ابعاد كلى \\
\hline
\end{tabular}

识

جدول r. ارتباط كيفيت زندكى با متغيرهاى جمعيتشناخى.

\begin{tabular}{|c|c|c|c|c|c|}
\hline P-value & ميانكين و انحراف معيار & متغير & P-value & ميانكين و انحراف معيار & متغير \\
\hline \multirow[b]{2}{*}{.180} & & شغل & & & تأهل \\
\hline & 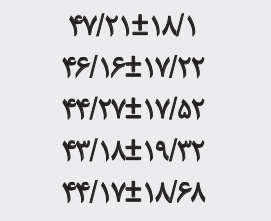 & خازئن بازئنداد & $.1 \cdot 1$ & 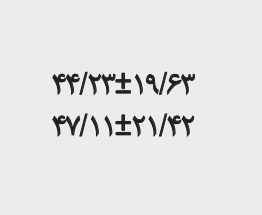 & مثأهل \\
\hline \multirow[b]{2}{*}{. $/ 4$} & & نوع درمان & & & جنسيت \\
\hline & 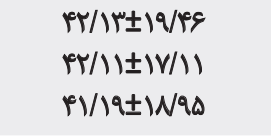 & هرو انسولين & $.1 .4 q$ & $\begin{array}{l}\text { FNIYIT./F } \\
R F / / 9 \pm I F / F\end{array}$ & هرد \\
\hline \multirow[b]{2}{*}{$\leq .1+.1$} & & درأمد (هزار تومان) & & & تحصيلات \\
\hline & 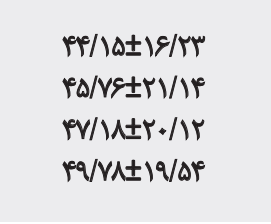 & 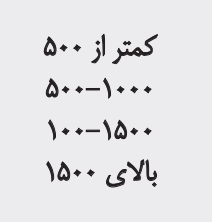 & .1 .14 & 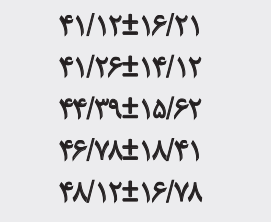 & 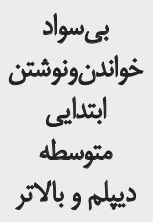 \\
\hline \multirow[b]{2}{*}{$\leq .1 \cdot+1$} & & عوارض هزمن ديابت & & & هرل سكونت \\
\hline & $\begin{array}{l}\text { PA/FAIWNTY } \\
\text { FNITITI/IF }\end{array}$ & ندارد & $1 \cdot n^{e}$ & $\begin{array}{l}P e / T I \\
P F / R T\end{array}$ & شهير \\
\hline
\end{tabular}

iU

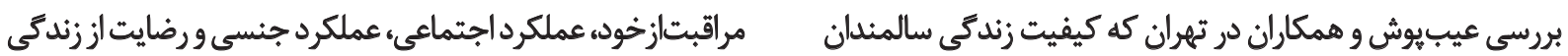

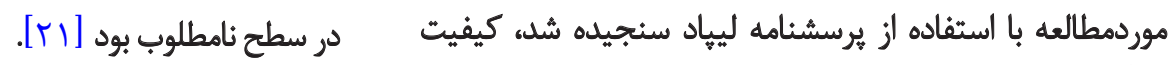

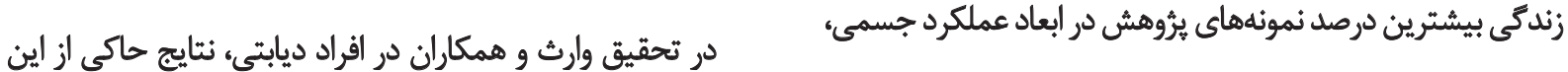


جدول P. ضرايب همبستكى بين كيفيت زندكى با سن، مدت ابتلا و شاخص توده بدنى.

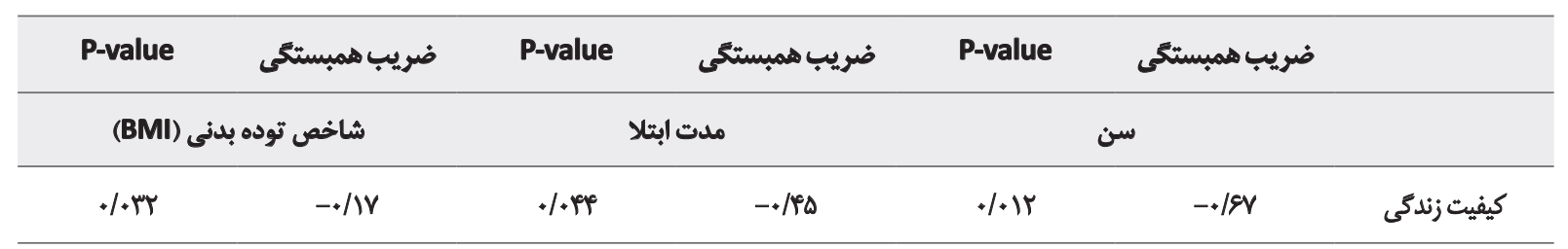

L

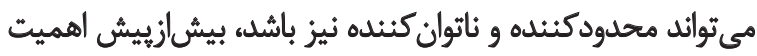

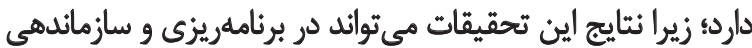

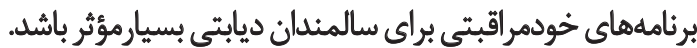
اكرجه عوامل متعددى با كيفيت زندگى در ارتباط هستند،

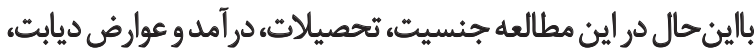

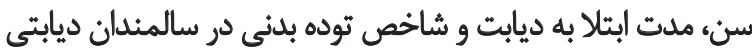

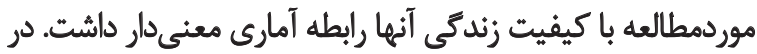

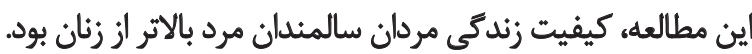
نتايج وحدانينيا و همجنين رفعتى در سالمندان تهرانى و مطالعهانيا

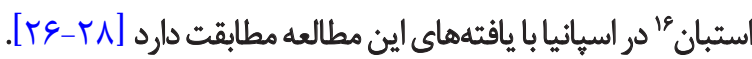

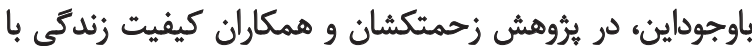

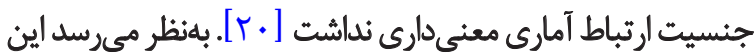

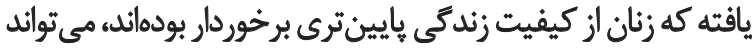

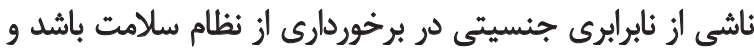

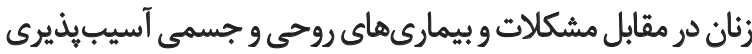

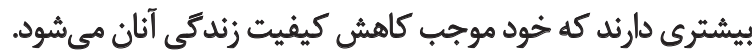
بين تحصيلات سالمندان ديابتى و كيفيت زندكى آنها نيز تفاوت

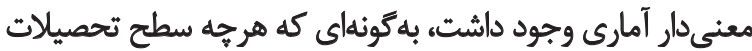

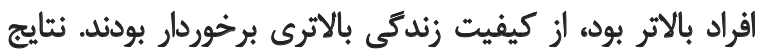

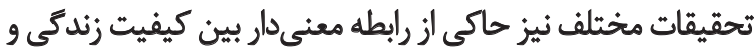

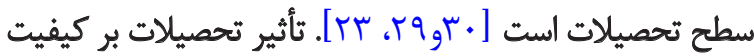

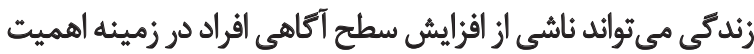

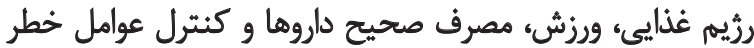

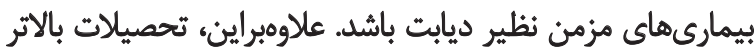

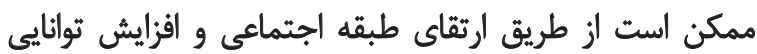

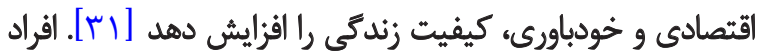

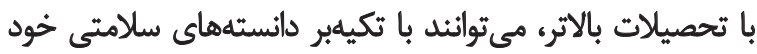

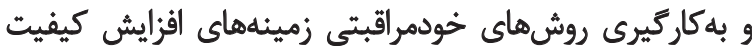

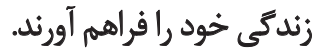

درآمد نيز يكى ديخر از عوامل مرتبط با كيفيت زندكى سالمندان

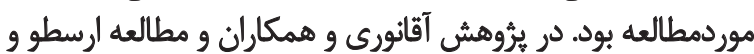

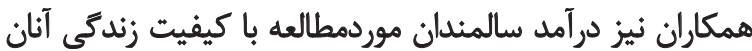

16. Esteban

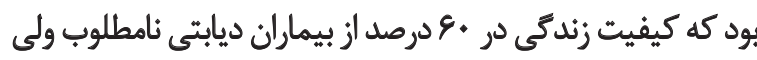

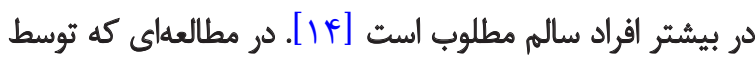

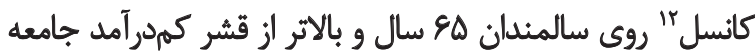

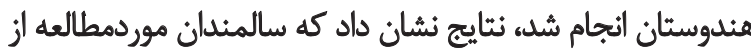

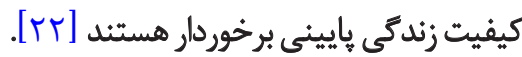

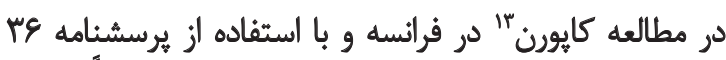

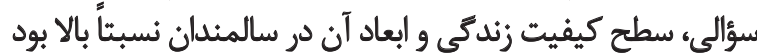

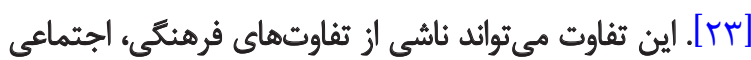

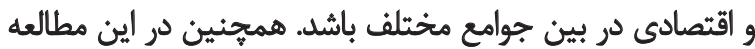

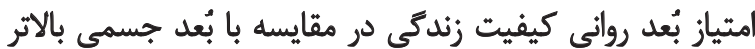

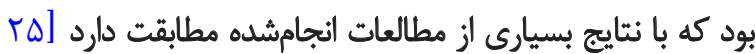

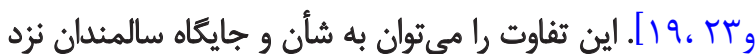

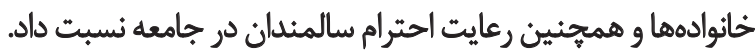

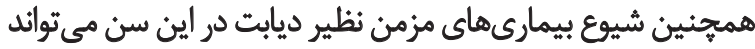

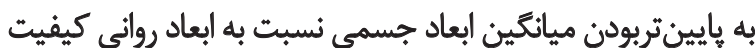

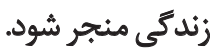

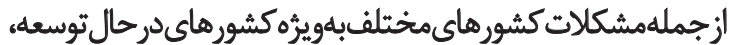

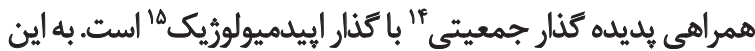

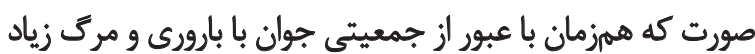

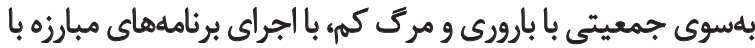

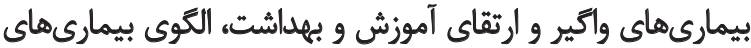
جامعه بهنفع بيمارى هاى مزمن مانند ديابت تغيير يافته است

در اين بين، مطالعات مختلف حاكى از آن است كه تعداد زيادى از ماز

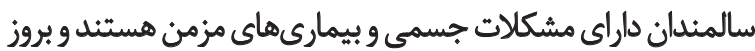

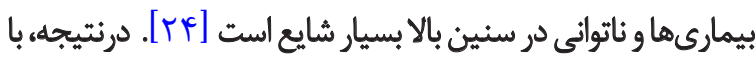

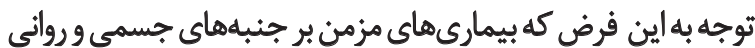

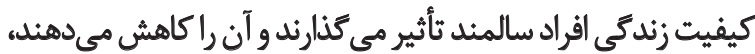

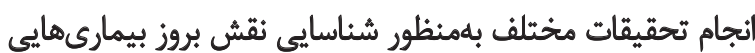

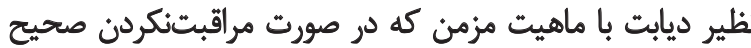

\section{Counsell}

13. Capuron

14. Demographic transition

15.Epidemiologic transition 


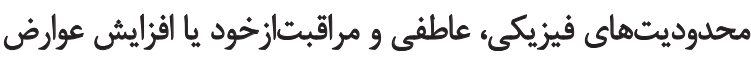

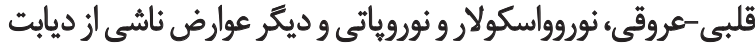

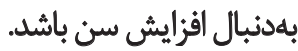

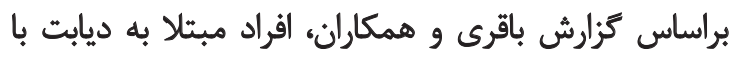

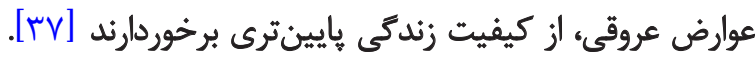

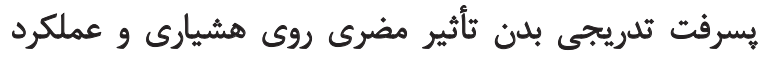

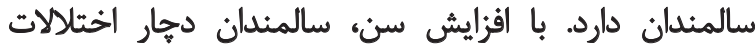

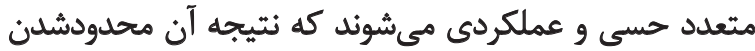

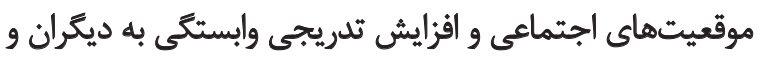

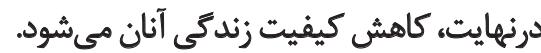

بين مدت ابتلا به ديابت و كيفيت زندكى بيماران ديابتى نيز

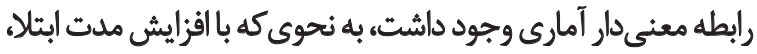

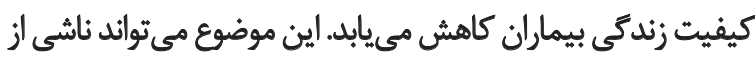

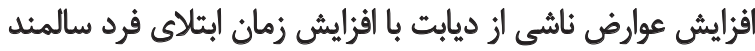

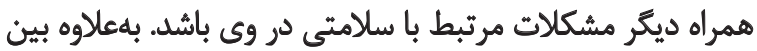

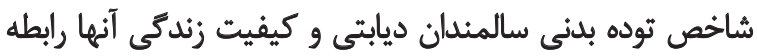

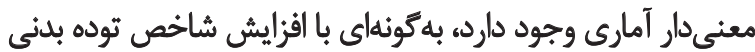
سالمندان، كيفيت زندكى آنها كاهش مي يابد باند.

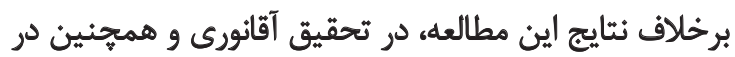

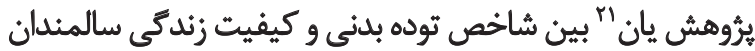

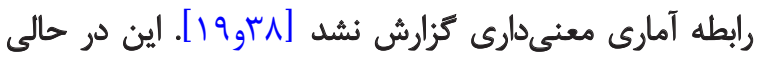

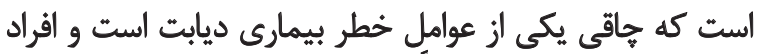

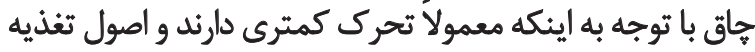

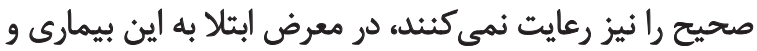

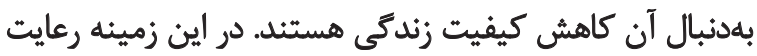

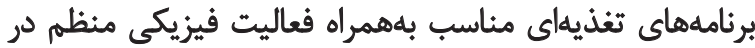
سنين سالمندى توصيه مي تشودي

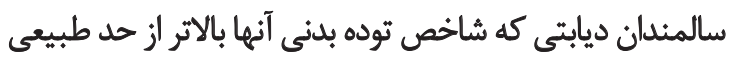

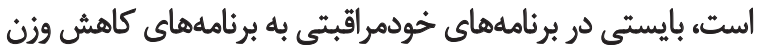

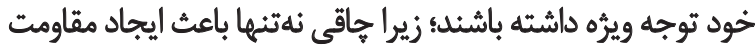

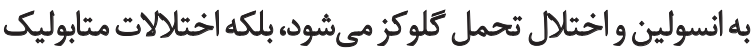

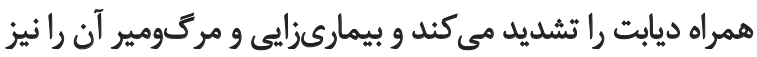

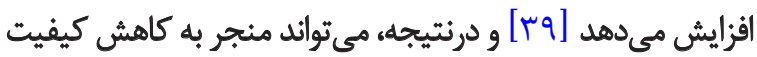

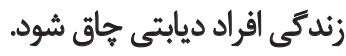

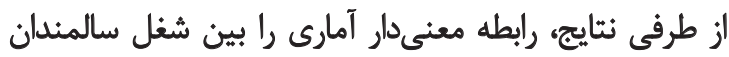

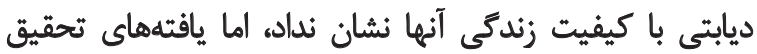

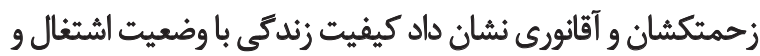
وضعيت اقتصادى ارتباط آمارى معنى دار دارد كه بانيت بانتايج اين مطالعيد

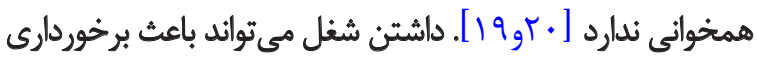

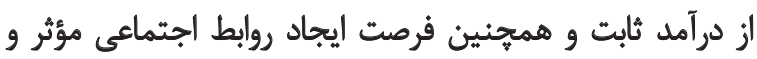

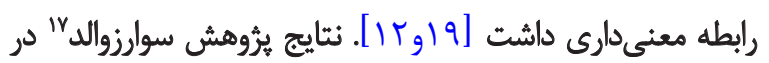

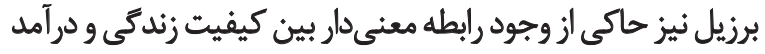

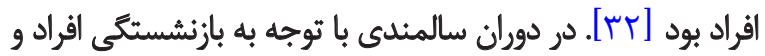

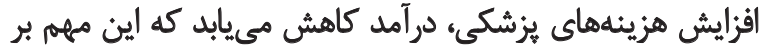
كيفيت زندكى آنان تأثير سوء دارد.

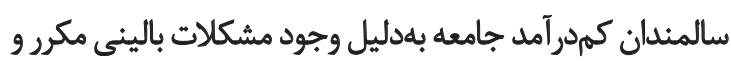

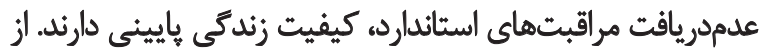

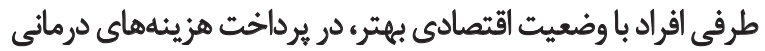

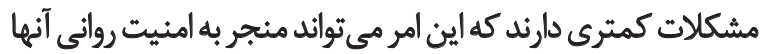

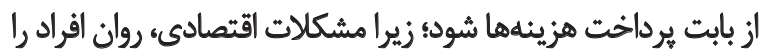

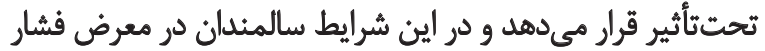

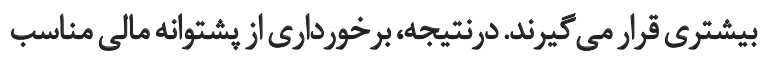
از عوامل مؤثر بر كيفيت زندكى آنها بهرئدمار مي آيد.

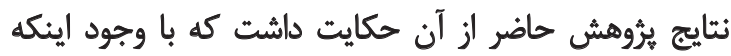

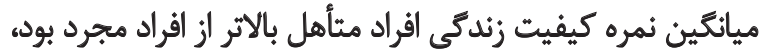

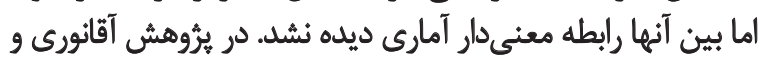

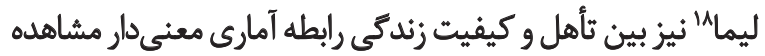

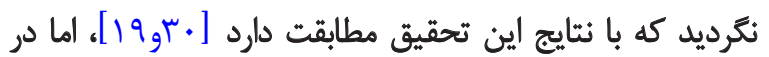

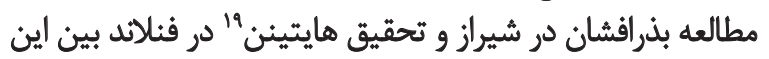

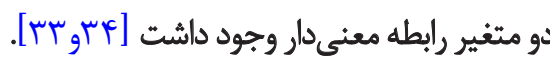

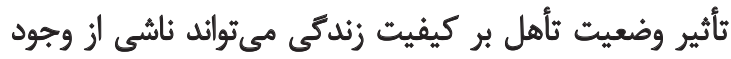

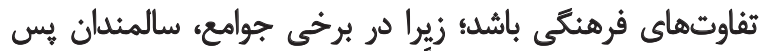

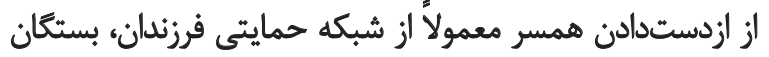

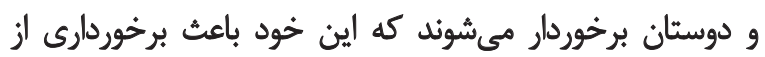

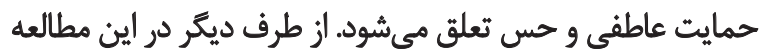

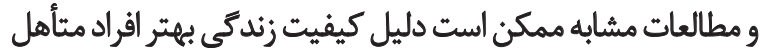

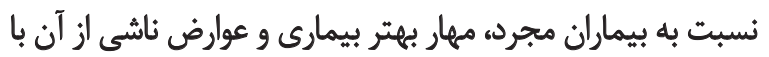

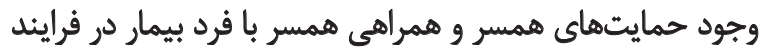

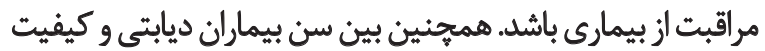

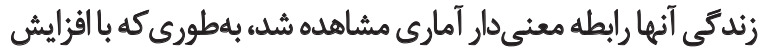
سن كيفيت زندگى بيماران كاهش مئسيابد

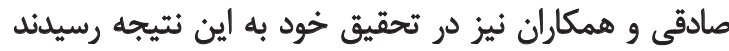

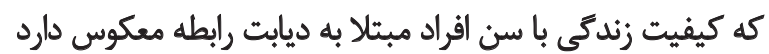

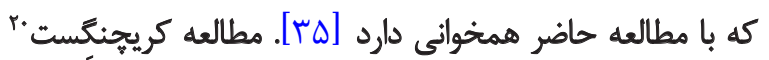

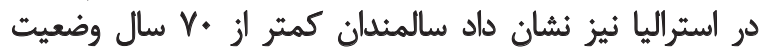

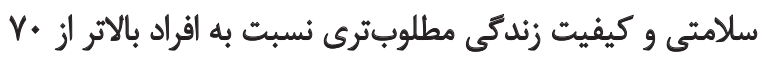

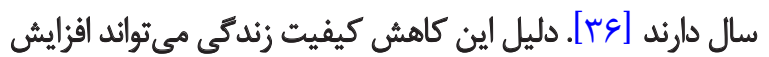

17. Szwarcwald

18. Lima

19. Hyttinen

20. Kirchengast 


$$
\text { تشكر و قدردانى }
$$

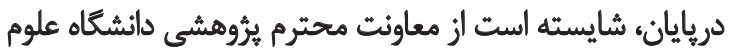

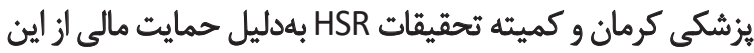

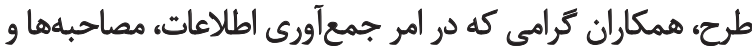

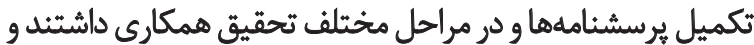

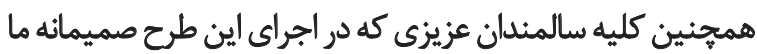
را همراهى نمودند، تشكر و قدردانى نماييم.
سازنده با افراد اجتماع شود و كيفيت زندكى را دهد.

ممكن است نبود رابطه بين دو متغير شغل و كيفيت به اين باين

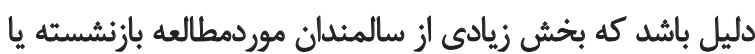

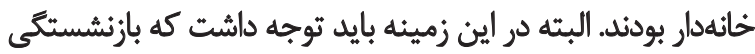

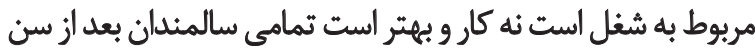

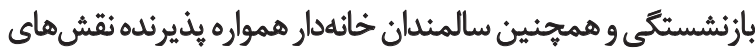

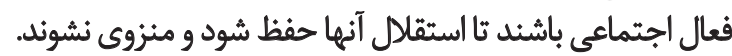

از طرفي بين محل سكونت بيماران ديابتى موردبررسي و كيفيت إنيت

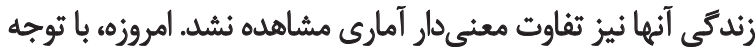

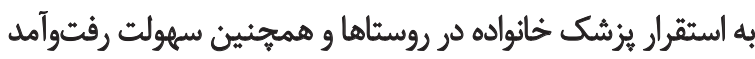

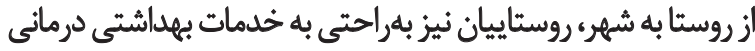

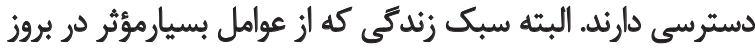

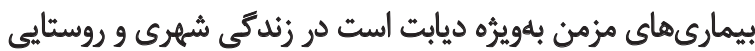

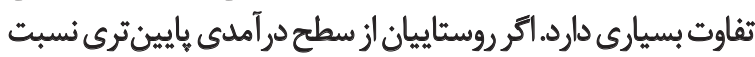
به شهرئشينان برخوردارند، در عوض سبك زندانيى سالمترى دارندي بين نوع درمان بيماران ديابتى موردبررسى و كيفيت زندكى آنها

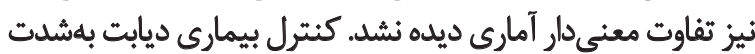

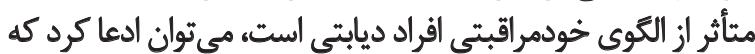

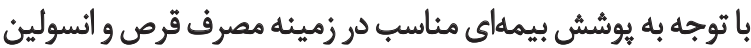

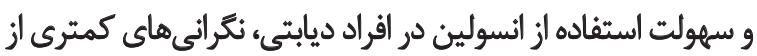
طرف افراد ديابتى در مورد درمان وجود دارداد.

\section{نتيجلة تيرىنهايى}

براساس يافتههاي اين تحقيق مبنىبر يايينبودن كيفيت زندكّى

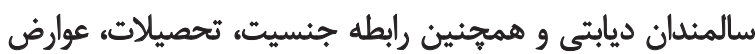

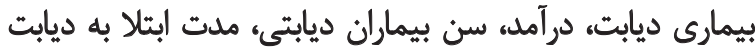

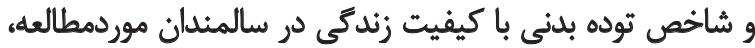

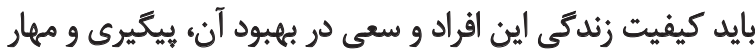

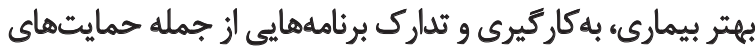
اجتماعى و اقتصادى و فراهمزنمودن شرايطى كه سالمندان نيازئهاي

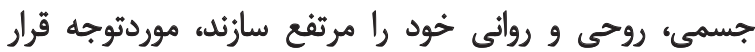

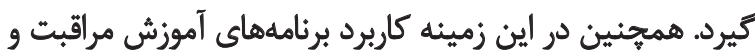

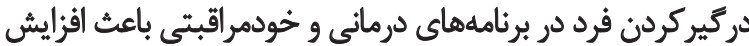
توان مراقبتى و بهبود كيفيت زند دركى آنان مي شودود.

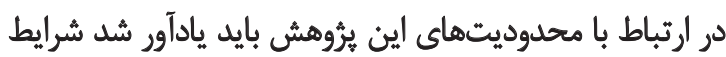

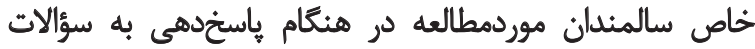

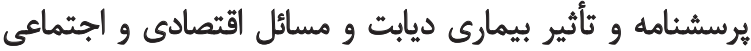

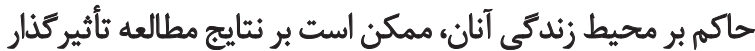

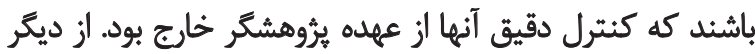

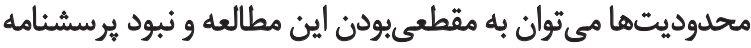
كيفيت زندگى خاص سالمندان ديابتى اشاره نمود. 


\section{References}

[1] Kinsella K, Phillips DR. Global aging: The challenge of success. population bulletin. Population Reference Bureau. 2005; 60(1):1-5.

[2] Newman AB, Murabito JM. The Epidemiology of Longevity and Exceptional Survival. Epidemiologic Reviews. 2013; 35(1):181-197.

[3] Ian SM, James RG, Beverly MS, Warwick R, Jill S, Dhigna R, Karen L. A cost effectiveness study of integrated care in health services delivery: A diabetes program in Australia. BMC Health Services Research. 2008; 8(1):201-5.

[4] Roith D, Taylor S, Olefsky J. Diabetes mellitus: A fundamental and clinical text. $3^{\text {rd }}$ ed. Philadelphia: Lippincott Williams \& Wilkins; 2004.

[5] Amini M, Parvaresh E. Prevalence of macroyan microvascular complications among patients with type 2 diabetes in Iran: A systematic review. Diabetes Research and Clinical Practice. 2009; 83(1):18-25.

[6] Ghanbari A, Parsa Yekta Z, Atrkar R. [The determine of effective factors on the quality of the lie in diabetic patients (Persian)]. Journal of Gillan University of Medical Sciences. 2001; 10(37-38):82-89.

[7] Wee HL, Cheung YB, Li SC, Fong KY, Thumboo J. The impact of diabetes mellitus and other chronic medical conditions on healthrelated Quality of Life: Is the whole greater than the sum of its parts? Health and Quality of Life Outcomes. 2005; 3(1):2.

[8] Sadeghi Ahari S, Arshi S, Iran Parvar M, Amani F, Seiah Posh H. [The effect of complications of diabetes Type II on the quality of life in diabetic patients (Persian)]. Journal of Ardebil University of Medical Sciences. 2008; 8(4):394-402.

[9] Leininger M. Quality of life from a Transcultural cultural nursing perspective. Nursing Science Quarterly. 2002; 7(1):22-28.

[10] Donald A. What is quality of life? $1^{\text {st }}$ ed. Hayward: Hayward Medical Communications; 1998.

[11] Ghafari Gh, Karimi A, Nozari H. [Trend study of quality of life in Iran (Persian)]. Quarterly of Social Studies and Research. 2012; 1(3):107-134.

[12] Arastoo A, Ghasemzade R, Nasseh H, Kamali M, Rahimi Foroshani A, Arzaghi S, et al. [Factors affecting quality of life in elderly diabetic residents of Kahrizak Geriatric Nursing Home of Tehran (Persian)]. Iranian Journal of Endocrinology and Metabolism. 2012; 14(1):18-24.

[13] Nejati V, Ashayeri H. [Health related quality of life in the elderly in kashan (Persian)]. Iranian Journal of Psychiatry and Clinical Psychology. 2008; 14(1):56-61.

[14] Vares Z, Zandi M, Baghaei P, Masoudi Alavi N, Mirbagher Ajorpaz N. [Study of quality of life and associated factors in diabetes mellitus patients of Kashan Diabet Center (Persian)]. Iranian Journal of Nursing Research. 2010; 5(17):14-22.

[15] Minet L, Mohler S, Vach W, Wagner L, Henriksen J. Mediating the effect of self-care management intervention in type 2 diabetes: A meta-analysis of 47 randomized controlled trials. Patient Educ ation and Counseling. 2010; 80(1):29-41.

[16] Hadipour M, Abolhasani F, Molavi-e Vardanjani H. [Health related quality of life in patients with of type II diabetes in Iran (Persian)]. Payesh. 2013; 12(1):135.141.
[17] Montazeri A, Goshtasebi A, Vahdaninia M, Gandek B. The short form health survey (SF-36): Translation and validation study of Iranian version. Quality of Life Research. 2005; 14(3):875-82.

[18] Harris MI, Flegal KM, Cowie CC, Eberhardt MS, Goldstein DE, Little RR, et al. Prevalence of diabetes, impaired fasting glucose, and impaired glucose tolerance in US adults: the Third National Health and Nutrition Examination Survey, 1988-1994. Diabetes Care. 1998; 21(4):518-24.

[19] Aghanoori A, Mahmoodi M, Salehi H, Jaferian K. [Quality of life in elderly health centers in urban areas in Markazi Province (Persian)]. Iranian Journal of Ageing. 2011; 6(4):21-29.

[20] Zahmatkeshan N, Bagherzade R, Akaberiyan SH, Yazdankhah Fard M, Mirzaei K, Yazdanpanah S. Assessing quality of life and related factors in Bushehr's elderly people (Persian)]. Journal of Fasa University of Medical Sciences. 2012; 2(1):53-58.

[21] Eybpoosh S, Aliasquarpoor M, Eybpoosh S, Aliasquarpoor M. [The Quality of life of elderly nursing Home residents and its relationship with different factors (Persian)]. Iran Journal of Nursing. 2012; 25(75):60-70.

[22] Counsell SR, Callahan CM, Clark DO, Buttar AB, Stump TE, Ricketts GD. Geriatric care management for low-income seniors: A randomized controlled trial. Journal of American Medical Association. 2007; 298(22):2623-3.

[23] Capuron L, Moranis A, Combe N, Cousson-Gelie F, Fuchs D, De Smedt-Peyrusse V. Vitamin E status and quality of life in the elderly: Influence of inflammatory processes. British Journal of Nutrition. 2009; 102(10):1390-4.

[24] Joghatai MT, Asadi MH. [The state of elderly people and the necessity of paying attention to their needs (Persian)]. Hakim. 2008; 1(2):6-11.

[25] Abbasi Moghadam MA, Dabiran S, Safdari R, Djafarian K. Quality of life and its relation to sociodemographic factors among elderly people living in Tehran. Geriatrics and Gerontology. 2009; 9(3):270-5.

[26] Ahangari M, Kamali M, Hesabi A. [Effect of hypertension on quality of life in members of Tehran senile cultural house club (Persian)]. Iranian Journal of Ageing. 2008; 3(7):26-32.

[27] Alipoor F, Sajadi H, Foroozan A, Biglarian A, Jalilian A. Quality of life of elderly regional $2^{\text {nd }}$ Tehran (Persian)]. Iranian Journal of Ageing. 2008; 3(9-10):75-83.

[28] Esteban Pena MM, Hernandez Barrera V, Fernandez Cordero X, Gil de Miguel A, Rodríguez Pérez M, Lopez de Andres A, et al. Self-perception of healthstatus, mental health and quality of life among adultswith diabetes residing in a metropolitan area. Diabetes Metabolism. 2010; 36(4):305-11.

[29] Aghamolaei T, Tavafian SS, Zare S. Health related quality of life in elderly people living in Bandar Abbas, Iran: A populationbased study. Acta Medica Iranica. 2010; 48(3):185-91.

[30] Lima MG, Barros MB, César CLG, Goldbaum M, Carandina L, Ciconelli RM. Health related quality of life among the elderly: A population-based study using SF-36 survey. Cadernos de Saúde Pública. 2009; 25(10):2159-67.

[31] Alexandre Tda S, Cordeiro RC, Ramos LR. Factors associated to quality of life in active elderly. Revista de Saúde Pública. 2009; 43(4):613-21. 
[32] Szwarcwald CL, Souza-Júnior PRB, Esteves MAP, Damacena GN, Viacava F. Socio-demographic determinants of selfrated health in Brazil. Cadernos de Saúde Pública. 2005; 21(1):54-64.

[33] Bazrafshan MR, Hosseini MA, rahgozar M, Sadat Maddah SB [Quality of elderly's life in Shiraz, Jahandidegan club (Persian)]. Iranian Journal of Ageing. 2008; 3(1):33-41.

[34] Hyttinen L, Kekalainen P, Vuorio AF, Sintonen H, Strandberg TE. Health-related quality of life in elderly patients with familial hypercholesterolemia. International Journal of Technology and Assessment Health Care. 2008; 24(2):228-34.

[35] Sadeghi Ahari S, Arshi S, Iranparvar Alamdari M, Amani F, Siahpoush $\mathrm{H}$. The effect of complications of type II diabetes on patients' quality of life (Persian)]. Journal of Ardabil University of Medical Sciences. 2009; 8(4):394-402.

[36] Kirchengast S, Haslinger B. Gender differences in health-related quality of life among healthy aged and old-aged Austrians: cross-sectional analysis. Gender Medicine. 2008; 5(3):270-8.

[37] Bagheri H, Ebrahimi H, Taghavi N, Hasani M. [Evaluation of quality of life in patients with diabetes mellitus, based on its complications, referred to Emam Hossein Hospital, Shahroud (Persian)]. Journal of Shahrekord University of Medical Sciences. 2005; 7(2):50-56.

[38] Yan LL, Daviglus ML, Liu K, Pirzada A, Garside DB, Schiffer L, et al. BMI and health-related quality of life in adults 65 years and older. Obesity Research. 2004; 12(1):69-76.

[39] Maggio CA. Obesity and type 2 diabetes. Endocrinology Metabolism Clinics of North America. 2003; 32:805-22. 
